\title{
Theoretical Model of the Probability of Fusion between Deuterons within Deformed Lattices with Micro-cracks at Room Temperature
}

\author{
Frisone Fulvio \\ Department of Physics, University of Catania, Via Santa Sofia 64, Catania 95125, Italy
}

Received: September 28, 2016 / Accepted: October 13, 2016 / Published: January 31, 2017.

\begin{abstract}
In this work, we wish to demonstrate that a reaction path as the following, dislocations, deformations due to thermodynamic stress and, finally, micro-crack occurrence, can enhance the process of fusion of the deuterons introduced into the lattice by deuterium loading. In fact, calculating the rate of deuteron-plasmon-deuteron fusion within a micro-crack, showed, together with an enhancement of the tunneling effect, an increase of at least 2-3 orders of magnitude compared to the probability of fusion on the no deformed lattice. In fact, strong electric fields can take place in the micro-crack and the deuterons are accelerated to the energy which are enough for the D-D tunnelling. These phenomena open the way to the theoretical hypothesis that a kind of chain reaction, catalyzed by the micro-cracks produced in the structure as a result of deuterium loading, can favour the process of deuteron-plasmon fusion.
\end{abstract}

Key words: Condensed matter, dislocations, lattice deformation, micro-cracks.

\section{Introduction}

In a previous paper [1], we studied the influence of the temperature on the phenomenon of deuteron fusion within unperturbed crystalline lattices with CFC (face centered cubic) or HCP (hexagonal centra face) structures, hypothesizing that a kind of chain reaction, catalyzed by the micro-cracks which form following ionic dislocations induced by structural deformations as a result of variations in the thermodynamic conditions or other factors, can increase the rate of fusion.

As a result of the numerical calculation performed for different metals, varying the temperature, the total energy and the concentration of impurities, it was possible to conclude that the probability of fusion was in effect substantially enhanced by increasing these

Corresponding author: Frisone Fulvio, nuclear researcher, research fields: theoretical physics, nuclear physics, low energy nuclear reactions, structure of matter, condensed matter science of metals. parameters.

In this paper we wish to analyze the role of impurities on dislocation formation and then on micro-crack occurrence. In fact within micro-crack local electrical field taking place is able to accelerate the deuterons along the coulomb barrier. It is important to observe that the micro-crack formation is a non-linear phenomenon. It can depend on impurities percentage, ratio loading, and other thermodynamic conditions. So from a point of experimental view, we can expect that the micro-crack formation is a very random effect. However, this is a very crucial point, the $\mathrm{D}-\mathrm{D}$ fusion enhancement within micro-crack (due to electrical local field) is a very linear effect.

\section{Lattice Screening Role}

After the Fleischmann and Pons' experiments [11] had been published, it soon became clear that the main role of Palladium lattice is catalysing. Many people 
supposed that in the lattice the coulomb potential is screened. To illustrate this topic we report the argument of Horowitz [4].

The electrons in a metal should become a Fermi gas and the hydrogen nuclei interacting via screened coulomb potential. The effective potential between two nuclei $V(r)$ which includes the effects of electron screening is given, in a simple Thomas-Fermi model, by:

$$
V(r)=\frac{e^{2}}{r} \exp \left[-\frac{r}{\lambda}\right]
$$

of course $\lambda$ is the screening length and depends on density. But for $r<<\lambda$, we can write at first one:

$$
V(r)=\frac{e^{2}}{r}-V_{0}
$$

this constant would be just the difference between electronic energy of a $\mathrm{He}$ isolated atom $(-79.0 \mathrm{eV})$ and the binding energy of two $\mathrm{H}$ atoms $(-51.8 \mathrm{eV})$. The fusion rate has been evaluated using:

$$
A=v P_{n}
$$

where, $v$ is the vibrational frequency of the crystal's zero point motion ( $h v$ is about $1 \mathrm{eV}$ ) and $P_{n}$ is the probability of a D-D nuclear reaction once the nuclei have made it to $r_{n}$. In other words the fusion rate is calculated by multiplying $P$ by the frequency of attacks on the coulomb barrier and the probability of a nuclear reaction.

Put $h v=1 \mathrm{eV}$ and $P_{n}=1$ (for D-D reaction), Horowitz computes $\Lambda=10^{-70} \mathrm{sec}^{-1}$, for $r_{0}=0.5 \AA$, but $\Lambda=10^{-25} \sec ^{-1}$ for $r_{0}=0.1 \AA$.

Similar argumentations were proposed by Giuliano Preparata but starting from a new formulation of condensed matter theory known as Coherence Theory. In fact according to the Coherence Theory of Condensed Matter the d-shell electrons screen the Coulomb potential and it is possible to demonstrate that:

$$
V(r)=\frac{e^{2}}{r}-85 e V
$$

It means that the deuterons can reach the intermolecular distance of $0.165 \AA$. In this case by means of Eq. (1) and using $A=10^{22} \sec ^{-1}$, he obtains $\Lambda=10^{-22} \sec ^{-1}$.

We conclude this section observing that within a lattice according to the quantum mechanics principles the fusion probability is observable.

\section{An Effective Potential Proposed}

From these results it appears clear that within lattice the $\mathrm{D}$-D reactions take place in a $\mathrm{D}_{2}$-molecule whose inter-nuclear distance is reduced by screening effect. More exactly by means of works reported in Refs. [2-12], we can say that within a lattice: the screening effect is able to increase the fusion rate a compound potential as which used by Ref. [5] is also able to reproduce the D-D interaction.

For this reason we tried to find a D-D effective potential such that for distance smaller than $\rho$ gives about the coulomb potential while, for the distance bigger than $\rho$, a Morse-like potential. In Ref. [1] to fit a such "Coulomb-Morse linked" potential we have proposed the following effective potential:

$$
V(r)=k \frac{e^{2}}{r}\left(V_{M}(r)-\frac{A}{r}\right)
$$

where,

$$
V_{M}(r)=D^{\prime}\left[e^{-2 \gamma\left(r-r_{0}^{\prime}\right)}-2 e^{-\gamma\left(r-r_{0}^{\prime}\right)}\right] .
$$

Here $A, D^{\prime}, \quad \gamma$ and $r_{0}{ }^{\prime}$ are parameters to determinate by means of fitting (Table 1 ).

The potential screening $V_{0}$ value has been taken from Preparata's model (average: $85 \mathrm{eV}$ ) and it means that $\rho=26.9 / V_{0}$ is about $0.165 \AA$. Then, regarding the other main values (i.e. equilibrium distance $r_{0}$ and disassociation energy $D$ ) we have used the many-body theory approach.

Table 1 Parameters fitting.

\begin{tabular}{ll}
\hline$\rho=0.165 \AA$ & $A=0.0001$ \\
$r_{0}=0.35 \AA$ & $r_{0}^{\prime}=0.99$ \\
$D=50 \mathrm{eV}$ & $D^{\prime}=1.49$ \\
& $\gamma=1.04$ \\
\hline
\end{tabular}




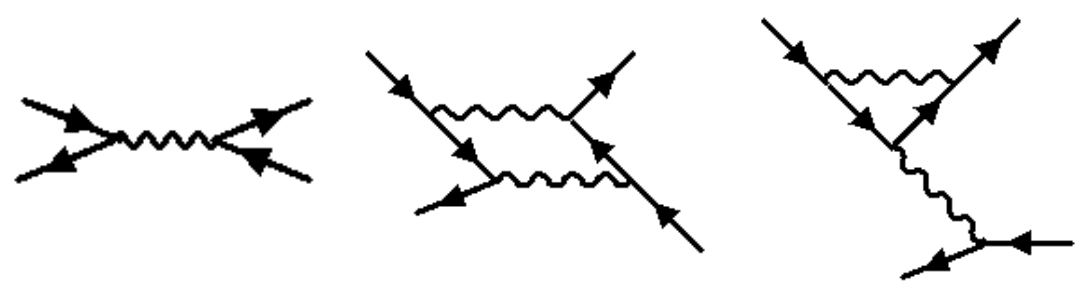

Fig. 1 Feymann expansion used.

In fact as pointed out in Ref. [11], the deuterons interaction with the collectives plasmon excitations of the palladium produces a strong attractive potential. This attractive force is due to exchange of plasmons (in Ref. [11] the authors consider only two plasmon excitations at $7.5 \mathrm{eV}$ and $26.5 \mathrm{eV}$ ) between two deuteron-lines as reported in Fig. 1.

Taking into account the role of coupling between deuteron and plasmons in the Ref. [6] is evaluated a like-Morse potential where $\rho$ is about $0.2 \AA$, D about $-50 \mathrm{eV}$ and $r_{0}$ about $1 \AA$.

Using the potential we obtain a fusion rate for $E=0$ of about $10^{-20} \sec ^{-1}$ (we used $A=10^{22} \sec ^{-1}$ ) (see Fig. 2).

\section{Deformation in Cubic Lattices}

In this section we wish to establish whether, and within what limits, the rate of fusion within a micro-crack in a generic cubic lattice subjected to deuterium loading can be conditioned or influenced, not only by extensive lattice defects and other characteristics and thermodynamic conditions, but also by any "deformations" produced in the crystalline lattice by variations in the temperature which finally can take place as micro-crack.

If this effectively occurs, it is not difficult to hypothesize that the energy produced by the micro-explosions within the micro-cracks present, could favor the creation of new fractures, which in turn would, by the same mechanism, capture other deuterons, and so on. On the other hand, the formation of micro-cracks in Palladium electrodes produced by the energy released during long periods of electrolysis

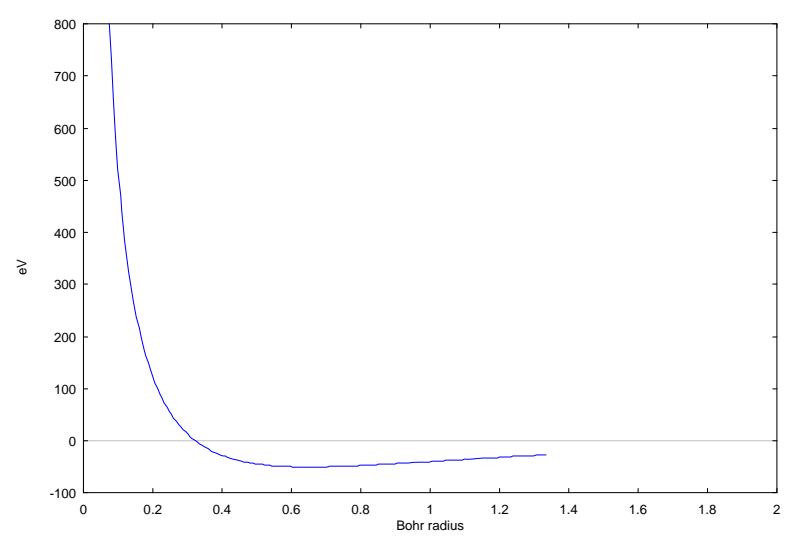

Fig. 2 Effective potential.

[12] has already been observed experimentally for some time but has until now been considered only a consequence of nuclear fusion. Our hypothesis is rather that this phenomenon could favor the process, enhancing the probability of fusion of the deuterons absorbed by the metal lattice.

Some particular reactions could then take place which incorporate the impurities in the nucleus of the dislocations [3], as a result of the different arrangement of the atoms with respect to that of the unperturbed lattice. An adequate theoretical description of the loading can therefore be obtained, in our opinion, only by treating it as a perturbation independent of time.

$$
\Psi=\Psi(J, r, R, \ldots)
$$

By the same way $J, r, R \ldots$ are correlated:

$$
R=R(r, J, \ldots)
$$

It means that the lattice loaded is a very complex system and then it exhibits a non linear behavior.

For simplicity we can now consider a cubic lattice structure subjected to deformations and calculate the probability of fusion within a micro-crack, $\Gamma$, on varying the temperature. 
Indicating the volume of a single cell by $d \Omega$, the deformation of the entire lattice is given by:

$$
\Psi \cong\left\{\iiint_{\Omega} \eta\left(J \frac{\rho L^{2} v \mathrm{~b}^{2}}{\alpha 2 h R} \chi \exp \left(-\frac{U_{0}}{k T}\right) \xi_{(r)}\right) d \Omega\right\}
$$

where, $\eta$ is a parameter which depends on the lattice and electronic structure of the metal under consideration.

In this study we have concentrated on the cubic structure of the lattice, in the specific case of Palladium, because it has an easily observable geometry and more exactly we have indicated by $\rho$ the density of the mobile dislocation [8] within the lattice at non constant lattice temperature, so that the thermodynamic stress of the deuterium nuclei for unit volume must be taken into consideration. Further, $L^{2}$ indicates the area between the lines of separation between two adjacent dislocations, produced during a deformation of the lattice [9] with non constant temperature, $R$ is the curvature which the dislocation assumes during deformation; $\alpha$ is proportional to the thermal increment and represents the effect of the sudden variations in temperature to which the lattice is subjected, as a result of which the deformations form; $v$ is the frequency of vibration of the deuterons in the metal, which we consider "negligible" here (and which will be considered at higher temperatures in future), $b^{2}$ is the stress line which dampens the transformation of the crystalline lattice for small stress variations within the lattice [9]; $U_{0}=2 U_{j}-\operatorname{Dbd}\left(\sigma-\sigma_{i}\right)_{\varepsilon_{0}}$ is the activation energy which is always less than a couple of jogs. In the latter expression, $\sigma-\sigma_{i}$ represents the stress applied at the small dislocations. Finally, $\xi_{(r)}$ is the number of dislocations.

We want to emphasize the deformation dependence on $\mathrm{k}$. In fact it is very easy to demonstrate that there exists a deuterium loading percentage (which at the same time depends on other thermodynamic conditions) $\chi_{0}$ for that happens

$$
\Psi \cong\left\{\iiint_{\Omega} \eta\left(J \frac{\rho L^{2} v \mathrm{~b}^{2}}{\alpha 2 h R} \chi_{0} \exp \left(-\frac{U_{0}}{k T}\right) \xi_{(r)}\right) d \Omega\right\} \geq p_{\text {lattice }}
$$

where, $p_{\text {lattice }}$ is the lattice pitch: in this case the micro-crack takes place.

Now we propose some approximate calculations in which the lattice deformation and the micro-crack depth are correlated. The depth of the micro-crack $L_{D}(T)$, a function of the lattice temperature, is given by Eq. (11):

$$
L_{D}(T)=\frac{F_{(o u t)} r}{J \xi_{(r)}\left[(l / 2)^{2}-r^{2}\right]^{1 / 2}}
$$

the quantity $\left(\frac{F_{(o u t)}}{L_{D}(T) J}\right) \delta r$ is the change in elastic and potential energies of the external mechanisms per unit of lattice length, due above all to the variations in the thermodynamic conditions caused by thermal exchange with the external system; $r$ is the distance between the center of the dislocation and the center of mass of the lattice, $\delta r$ is a small variation. The quantity:

$$
l=\frac{\mu N b}{\pi\left(1-\nu_{d}\right) J \sigma_{r}}
$$

represents the length of a micro-crack in a generic cubic lattice in conditions of quasi-equilibrium (the typical value [5] is $l \approx 700 \AA$ ), $\quad N b$ is the number of lattice ions, of mass $\mu$, involved in a super-dislocation (this number generally depends on the extension of the core). If the energy of the dislocation is limited by a cut-off $R$, within the metal there is a lattice configuration which is only slightly modified and whose particularities could depend on the external energy.

$v_{d}$ is the frequency of deformation:

$$
\boldsymbol{V}_{d}=\frac{2 W_{k}}{a^{2} k T} D_{k} \exp \left(-\frac{2 F_{k}}{k T J}\right)
$$


Eq. (13) represents the independence of the internal stress from the external conditions, a valid hypothesis in this approximation: $a^{2}$ is the position of equilibrium of the distribution core, separated along a "split" in the crystalline lattice of generic symmetry; $2 W_{k}$, approximately, is the energy of the barrier in different states of near equilibrium of the nuclei within the lattice, which includes the stress in conditions of non-equilibrium, $k T$ is the thermal energy to which the metal is subjected; $2 F_{k}$ is a parameter which depends on the lattice; $D_{k}$ represents the point of fusion with energy $k T$. Further, the factor $\sigma_{r}$ in Eq. (14), is the stress factor in quasi-equilibrium:

$$
\sigma_{r}=\frac{\mu b r}{2 \pi(1-v) D\left(r^{2}\right) J}
$$

where, $v$ is the frequency of the lattice vibrations induced by the dislocations, $D$ the distance between dislocations and $b$ the three-dimensional Burgers vector [7].

From this model the micro-crack formation appears around $300 \mathrm{~K}$ and the width fracture is $1-10 \mathrm{~nm}$.

\section{Conclusions}

The principal objective of the present study was to demonstrate if and how the deformation of the crystalline lattice and the formation of a micro-crack could influence the process of fusion at room temperature.

More precisely, we calculated numerically the probability of fusion within a micro-crack, comparing it with that calculated on the surface to evidence a possible enhancement effect.
Further study in this direction, and for other lattice structures, are in progress and the results obtained will be published in a future paper.

However, it is possible to confirm that the initial results obtained so far validate the hypothesis presented here.

\section{References}

[1] Frisone, F. 1998. Can Variations in Temperature Influence Deuteron Interaction within Crystalline Lattices? 1567-80.

[2] Rabinowitz, M. 1990. "High Temperature Superconductivity and Cold Fusion.” Modern Physics Letters $B 4$ (4): 233-47.

[3] Hirt, J. P., and Lothe, J. 1960. Theory of Dislocation. McGraw Hill.

[4] Ashcroft, N. W., and Mermin, N. D. 1972. Solid State Physics. Philadelphia: Saunders College.

[5] Gutkin, M. Y., and Ovid'Ko, I. A. 1994. "Disclinations, Amorphization and Microcrack Generation at Grain Boundary Junctions in Polycrystalline Solids.” Philosophical Magazine A 70 (4): 561-75.

[6] Frisone, F. "Deuteron Interaction within a Microcrack in a Lattice at Room Temperature.” Date accepted for publication: 13/6/00, Fusion Tecnology.

[7] Hummel, R. E. 1997. Understanding Materials Science. Springer.

[8] Nabarro, F. R. N. 1952. Proceedings of the Royal Society of London. Series A 209, 219, Advances in Physics 1, 271.

[9] Friedel, J. 1960. Dislocations. Chapter V, 116-458.

[10] Sumino, K. 1988. "Point and Extended Defects in Semiconductors.” Nato ASI Series B 202: 77-83.

[11] Fleishmann, M., and Pons, S. 1989. "Electrochemically Induced Nuclear Fusion of Deuterium.” Journal of Electroanalytical Chemistry 261 (2): 301-8.

[12] An, H. K. et al. 1995. "Analysis of Deformed Palladium Cathodes Resulting from Heavy Water Electrolysis." Fusion Technology 27: 408-15. 